\title{
Fabrication, Characterization, and In Vivo Evaluation of Famotidine Loaded Solid Lipid Nanoparticles for Boosting Oral Bioavailability
}

\author{
Muhammad Shafique, ${ }^{1,2}$ Mir Azam Khan, ${ }^{1}$ Waheed S. Khan, ${ }^{2,3}$ Maqsood-ur-Rehman, ${ }^{1,2}$ \\ Waqar Ahmad, ${ }^{1}$ and Shahzeb Khan ${ }^{1}$ \\ ${ }^{1}$ Department of Pharmacy, University of Malakand, Chakdara, Dir (L), Khyber Pakhtunkhwa 18800, Pakistan \\ ${ }^{2}$ Nano-Biotech Group, National Institute for Biotechnology and Genetic Engineering, Faisalabad 38000, Pakistan \\ ${ }^{3}$ Ningbo Institute of Materials Technology and Engineering (NIMTE), Chinese Academy of Sciences (CAS), Ningbo, Zhejiang, China \\ Correspondence should be addressed to Mir Azam Khan; mirazam786@yahoo.com
}

Received 31 July 2017; Revised 14 November 2017; Accepted 20 November 2017; Published 14 December 2017

Academic Editor: Mohamed Bououdina

Copyright (C) 2017 Muhammad Shafique et al. This is an open access article distributed under the Creative Commons Attribution License, which permits unrestricted use, distribution, and reproduction in any medium, provided the original work is properly cited.

\begin{abstract}
Famotidine as $\mathrm{H}_{2}$ receptor has antagonistic effects on gastric secretion. Unfortunately, its hydrophobic nature contributes to its variable and poor oral bioavailability. In the current study efforts are being made to fabricate famotidine loaded solid lipid nanoparticles with narrow size distribution. Prepared nanoformulations were pharmaceutically evaluated to confirm the desired boosted oral bioavailability. Famotidine loaded nanoformulation (FFSe-4) showed particle size $111.9 \pm 1.3 \mathrm{~nm}$, polydispersity index $0.464 \pm 0.03$, zeta potential $-33.46 \pm 2 \mathrm{mV}$, entrapment efficiency $84 \pm 2.7 \%$, and drug loading capacity $2.709 \pm 0.13 \%$. Drugexcipients compatibility was confirmed by Fourier transformed infrared spectroscopy. Scanning electron microscopy confirmed spherical shaped, nanosized particles. Differential scanning calorimetry and powder X-ray diffractometry confirmed the change in crystalline nature. Prepared nanoformulation was more stable at refrigerated temperature. In vitro study showed that drug release time is proportional to drug pay load and followed zero order kinetics. Release exponent $(n>0.5)$ confirmed non-Fickian-diffusion mechanism for drug release. In vivo pharmacokinetic studies showed 2.06-fold increase in oral bioavailability of famotidine dispersed in solid lipid nanoparticles compared to commercial product. These results authenticate solid lipid nanoparticles as drug delivery system and propose prolonged release with improved oral bioavailability for famotidine.
\end{abstract}

\section{Introduction}

Approximately $40 \%$ of commercialized active pharmaceutical ingredients (APIs) are poorly water soluble, due to which sufficient amount of drug absorption from the gastrointestinal tract (GIT) is being a challenge for the researchers [1]. Low solubility and permeability lead to oral bioavailability issues which ultimately affect the drug safety and efficacy [2]. Previously, different colloidal carrier systems have been investigated to overcome this problem. But certain disadvantages were associated with them such as drug expulsion upon storage, limited stability, low drug loading, and polymers cytotoxicity [3].

This leads to the rise of fabricating solid lipid based nanodrug delivery system termed as solid lipid nanoparticle.
Solid lipid nanoparticles (SLNs) were developed in the end of the 20th century [4]. It potentially gathers pluses of the old systems but avoids some of their major documented shortcomings [5]. The use of SLNs is a striking improvement because the solid matrix of the lipids presents high flexibility in controlling the drug release and protects the encapsulated drugs from gastric degradation. SLNs are generally composed of biodegradable and biocompatible solid lipid as solid core, coated by nonhazardous surfactant/cosurfactant as the outer shell [6]. Use of solid lipids increases drug absorption mainly through enhanced drug dissolution and solubilization in the intestinal-milieu, improved lymphatic-transport, enhanced gastrointestinal permeability, and decreased gastric-emptying rate $[7,8]$. Particle size and PDI are key characteristics 
<smiles>NC(N)=Nc1nc(CSCC/C(N)=N/S(N)(=O)=O)cs1</smiles>

FIGURE 1: Chemical structure of famotidine.

and are critical parameters in the stability and fabrication of SLNs [9]. These characteristics mainly depend upon particles composition and different fabrication techniques.

Famotidine is widely used as competitive $\mathrm{H}_{2}$ receptor antagonist $\left(\mathrm{H}_{2} \mathrm{RA}\right)$ and prokinetic drug [10]. Molecular formula of famotidine is $\mathrm{C}_{8} \mathrm{H}_{15} \mathrm{~N}_{7} \mathrm{O}_{2} \mathrm{~S}_{3}$ and IUPAC name is 3-[[2-(diaminomethylideneamino)-1,3-thiazol-4-yl]methylsulfanyl]- $\mathrm{N}^{\prime}$ sulfamoylpropanimidamide (Figure 1). Its key pharmacodynamic effect is the inhibition of gastric acid secretion [11]. It decreases stomach acid production up to $90 \%$ when given in oral dosage form ( $20 \mathrm{mg}$ or $40 \mathrm{mg}$ ) and promotes duodenal ulcer curing [12]. It is used in the treatment of heart-burn, ulcer, and inflammation of esophagus and high doses are used for the treatment of conditions like ZollingerEllison syndrome. It is commercially available in different dosage forms like capsules, tablets, and also chewable tablets for adults. Powder was also prepared for oral suspension, but after reconstitution its stability was limited to thirty days only and also had extremely bitter taste [12]. Hence, researchers also tried numerous techniques to mask its bitter taste [13]. Hydrophobic nature of famotidine reduces its water solubility and also exposure to gastric degradation contributes to its variable and poor oral bioavailability [14].

Famotidine belongs to Class-IV drugs of biopharmaceutical classification system (BCS-IV). Drugs of this class show poor aqueous solubility and low permeability [15]. Due to which its oral formulations have not been successful due to low water solubility issues $\left(1.1 \mathrm{mg} \cdot \mathrm{ml}^{-1}\right)$ and unfavorable pharmacokinetic parameters, including low oral bioavailability $(43 \%)$ and a short plasma half-life (2.59 hrs) [1618]. Before selecting famotidine as drug model for loading into SLNs, the available limited literature for addressing the oral bioavailability issues has been studied. Patel, Dhaval J., et al. 2010 have reported FTD nanosuspension having minimum particle size of only $566 \mathrm{~nm}$ and also lacking stability study. Also, there has not been reported any in vivo pharmacokinetic study.

This research work was carried out to fabricate FTD loaded SLNs to enhance its aqueous solubility which in turn boost its oral bioavailability. SLNs were fabricated by solvent emulsification evaporation technique which is most suitable for of thermosensitive drugs as it avoids thermal stress [19]. SLNs have adhesive properties that could increase the residence time in the administered area and hence enhance its oral bioavailability [20]. The use of tween-80 as surfactant and PVP as cosurfactant may also improve oral bioavailability as they contribute to enhancing permeability as well as affinity between lipids and intestinal membrane [21, 22].
TABLE 1: Formulations of unloaded SLNs.

\begin{tabular}{lcccc}
\hline Formulation & $\begin{array}{c}\text { Stearic acid } \\
(\mathrm{g})\end{array}$ & $\begin{array}{c}\text { Tween-80 } \\
(\mathrm{ml})\end{array}$ & $\begin{array}{c}\text { PVP } \\
(\mathrm{g})\end{array}$ & $\begin{array}{c}\text { Stirring time } \\
(\mathrm{min})\end{array}$ \\
\hline UFSe-1 & 1.00 & 0.5 & Nil & 5 \\
UFSe-2 & 1.00 & 1 & Nil & 5 \\
UFSe-3 & 1.00 & 1.5 & Nil & 5 \\
UFSe-4 & 1.00 & 2 & Nil & 5 \\
UFSe-5 & 1.00 & 1.9 & 0.1 & 5 \\
UFSe-6 & 1.00 & 1.8 & 0.2 & 5 \\
UFSe-7 & 1.00 & 1.7 & 0.3 & 5 \\
UFSe-8 & 1.00 & 1.6 & 0.4 & 5 \\
UFSe-9 & 1.00 & 1.5 & 0.5 & 5 \\
UFSe-10 & 1.00 & 1.6 & 0.4 & 10 \\
UFSe-11 & 1.00 & 1.6 & 0.4 & 15 \\
UFSe-12 & 1.00 & 1.6 & 0.4 & 20 \\
\hline
\end{tabular}

PVP: polyvinyl pyrrolidone.

\section{Materials and Methods}

2.1. Materials. Famotidine was procured as generous gift from Polyfine Chempharma (Pvt) Ltd (Peshawar, Pakistan). Stearic acid and tween-80 were got from Acros Organics Thermo Fisher Scientific, New Jersey, USA. Polyvinylpyrrolidone (PVP-K30) was got from Crescent Chemical Company, Islandia, New York, USA. Dialysis bags were obtained from Spectrum lab Canada. Remaining materials were of analytical grade or equivalent.

\subsection{Methods}

2.2.1. Preparation of Unloaded SLNs. Unloaded SLNs were fabricated by solvent emulsification evaporation (SEE) technique, using different surfactant (tween-80) concentration, cosurfactant (PVP) concentration, and stirring time (Table 1) [19]. Specified amount of stearic acid was dissolved in chloroform which was then emulsified with aqueous phase having surfactant (Tween-80) and cosurfactant (PVP) under magnetic stirring (1000 rpm) to form microemulsion. In this microemulsion, aqueous phase contains micron-size droplets of organic solvent containing stearic acid. Organic solvent is evaporated from this microemulsion via magnetic stirring. As the organic solvent evaporates, the lipid starts precipitating as SLNs in the aqueous phase is followed by centrifugation using ultra-centrifuge Cs 150 GXL (GxSeries) for 10 minutes, at $30,000 \mathrm{rpm}$ [23]. $Z$-average particle size and PDI of these formulations were figured out by photon correlation spectroscopy using zeta-sizer Nano (ZS90, Malvern Instruments, Malvern, UK) [24].

2.2.2. Preparation of FTD-SLNs. Best conditions of UFSe-11 formulation, that is, stearic acid $(1.0 \mathrm{~g})$, tween-80 $(1.6 \mathrm{ml})$, and PVP $(0.4 \mathrm{~g})$, were further used for fabricating FTD loaded SLNs (FTD-SLNs). Different formulations of FTDSLNs were prepared on the basis of lipid drug ratio (Table 2). Specified quantity of FTD and stearic acid was dissolved in 


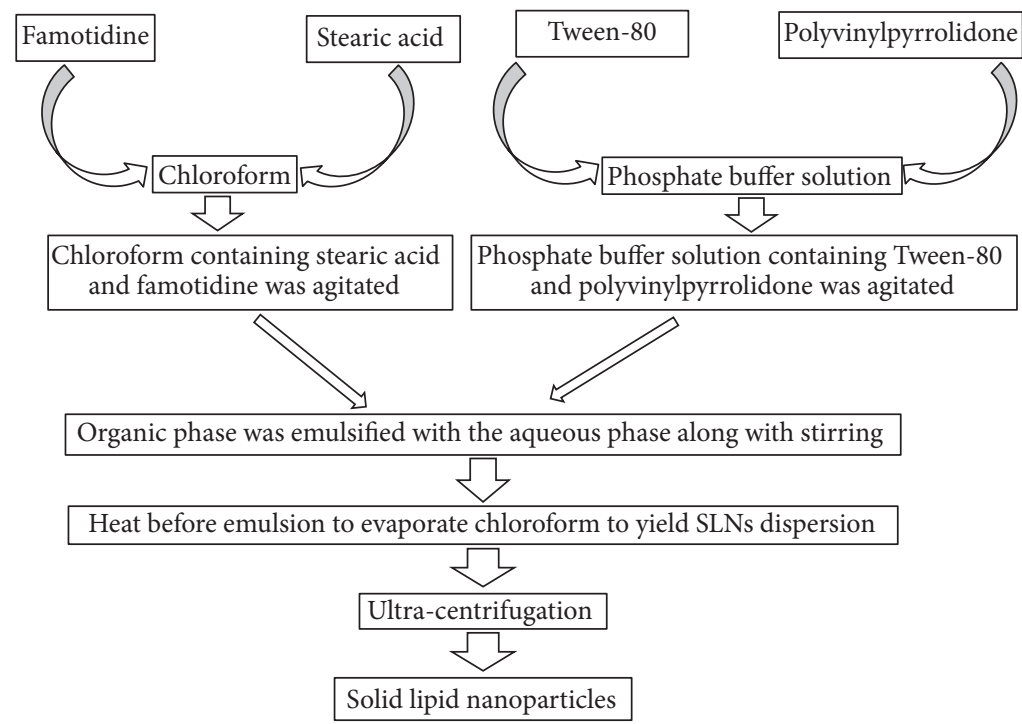

FIGURE 2: Schematic diagram of solvent emulsification evaporation technique.

TABLE 2: Formulations of FTD-SLNs.

\begin{tabular}{lccccc}
\hline Formulation & $\begin{array}{c}\text { Stearic acid } \\
(\mathrm{g})\end{array}$ & $\begin{array}{c}\text { FTD } \\
(\mathrm{mg})\end{array}$ & $\begin{array}{c}\text { Tween-80 } \\
(\mathrm{ml})\end{array}$ & $\begin{array}{c}\text { PVP } \\
(\mathrm{g})\end{array}$ & $\begin{array}{c}\text { Stirring time } \\
(\mathrm{min})\end{array}$ \\
\hline FFSe-1 & 1.00 & 40 & 1.6 & 0.4 & 15 \\
FFSe-2 & 1.00 & 50 & 1.6 & 0.4 & 15 \\
FFSe-3 & 1.00 & 66.6 & 1.6 & 0.4 & 15 \\
FFSe-4 & 1.00 & 100 & 1.6 & 0.4 & 15 \\
FFSe-5 & 1.00 & 200 & 1.6 & 0.4 & 15 \\
\hline
\end{tabular}

FTD: famotidine; PVP: polyvinyl pyrrolidone.

chloroform. The rest of process followed was same as adopted for unloaded SLNs. Schematic diagram for preparation of FTD-SLN is shown (Figure 2).

2.2.3. Lyophilization. SLNs are thermodynamically insecure systems; therefore, FTD-SLNs were lyophilized using freeze dryer (Heto Power Dry LL1500- Thermo Electron Corporation, USA). Glucose solution (10\%) was added as cryoprotectant before drying. FTD-SLNs were kept overnight at $-20^{\circ} \mathrm{C}$ and then shifted to freeze dryer to be lyophilized at $-75^{\circ} \mathrm{C}$ for $48 \mathrm{hrs}$ at increasing rate of $5^{\circ} \mathrm{C} / \mathrm{h}[25]$.

2.2.4. Entrapment Efficiency (EE) and Drug Loading Capacity $(D L C)$. Freshly fabricated FTD-SLNs samples were centrifuged and supernatants were analyzed to quantify unentrapped drug using nanodrop spectrophotometer (Thermo scientific 2000c/2000 UV-VIS Spectrophotometer).

Entrapment efficiency of FTD was calculated by

$\mathrm{EE} \%$

$=\frac{\text { Total amount of drug added }- \text { Unloaded Drug } \times 100}{\text { Total amount of drug added }}$.
Percent drug loading capacity of FTD was calculated by

$$
\text { DLC } \%=\frac{\text { Total amount of drug }(\text { SLNs }) \times 100}{\text { Amount of Drug }+ \text { Amount of Excipients }} .
$$

\subsection{Characterization}

2.3.1. Dynamic Light Scattering. Zeta-sizer analysis was carried out by using zeta-sizer ZS-90 (Malvern Instruments, England). $Z$-average particle size, PDI, and zeta potential were analyzed. All SLN formulations were diluted with deionized water in order to get proper scattering intensity, measured at $90^{\circ}$ scattering angle and $25^{\circ} \mathrm{C}$.

2.3.2. Drug-Excipients Interaction. Fourier transform infrared spectroscopy (IR Prestige 21 Shimadzu, Japan) was used to study drug-excipients interaction with diffuse reflectance principle [26]. Spectra of unprocessed FTD and processed FTD (FFSe-4) were scanned over a frequency range of 2000 to $400 \mathrm{~cm}^{-1}$. For compatibility of formulation components, the peaks and patterns shaped by the unprocessed FTD were compared with processed FTD (FFSe-4).

2.3.3. Morphological Study. Scanning electron microscopy (SEM) was used to study the morphological characteristics and texture of SLNs by JSM5910 (JEOL, Japan) [27]. SEM micrographs were recorded at magnification of 60,000x and accelerating voltage of $20 \mathrm{kV}$ [28].

2.3.4. Powder X-Ray Diffraction (P-XRD). Powder X-ray diffraction analysis was performed to verify new solid state formation [29]. P-XRD analysis was conducted for unprocessed FTD and processed FTD (FFSe-4) using an X-ray diffractometer JDX-3532 (JEOL Japan). $\mathrm{Cu} \mathrm{K} \alpha$ radiation in scanning range of $2 \theta=5^{\circ}-80^{\circ}$ was used with tube current $30 \mathrm{~mA}$, operated voltage of $40 \mathrm{kV}$, step time $1.0 \mathrm{sec}$, step size 
$0.05^{\circ}$, divergence slit 1 degree, scattering slit 1.0 degree, and receiving slit $0.2 \mathrm{~mm}$ for measurement.

2.3.5. Thermal Analysis. Differential scanning calorimetry (DSC) is thermoanalytical method used to investigate melting and recrystallization behavior of samples. Accurately weighted unprocessed FTD, stearic acid, their physical mixture, and processed FTD (FFSe-4) were analyzed by differential scanning calorimeter (DSC) (Perkin Elmer, Diamond Series DSC Equipment-USA). Analyses were carried out in crimped aluminum pans at heating rate of $10^{\circ} \mathrm{C} / \mathrm{min}$ from $40-300^{\circ} \mathrm{C}[30]$.

2.4. Stability Study. Stability study was conducted at various temperatures in terms of measurement of particle size and PDI with respect to time.

To examine the physical stability of FTD-SLNs, stability study was carried out for FFSe-4 formulation [31]. The freshly fabricated sample was divided into two parts. Each part was put in two plain sealed glass vials and stored at different temperatures $\left(5 \pm 2^{\circ} \mathrm{C}\right.$ and $\left.25 \pm 3^{\circ} \mathrm{C}\right)$ for 3 months. Samples were taken on 1st, 15th, 30th, 60th, and 90th day of storage and subjected to particle size and PDI measurements. Data was analyzed statistically by two tailed $t$-test. Probability < 0.05 was considered significant.

2.5. In Vitro Release of FTD from SLNs. In vitro drug release study was conducted using dialysis bag method [32]. Dialysis bags were soaked in deionized water for 12 hours before use. FTD-SLNs dispersion $(1 \mathrm{ml})$ from each formulation was poured into the dialysis bag and placed in $250 \mathrm{ml}$ phosphate buffer solution ( $\mathrm{pH} 7.4$ ) at $50 \mathrm{rpm}$. After definite time interval (1-12 hr), samples were taken and equal volume of phosphate buffer solution was replaced. Samples were analyzed by using UV spectrophotometer $\left(\lambda_{\max } 265 \mathrm{~nm}\right)$ against blank phosphate buffer solution (pH 7.4) [33]. Data obtained from in vitro drug release study was fitted into different kinetic models to find out both drug release rate and mechanism that followed [34].

\subsection{In Vivo Pharmacokinetic Studies}

2.6.1. Oral Drug Administration. Before conducting in vivo study, approval was taken from departmental research ethics committee (vide letter number DREC/20160503-14). Healthy rabbits $(2 \pm 0.3 \mathrm{Kg})$ were kept fasted $(12 \mathrm{hrs})$ before dosing but access to water was given. Two groups of animals were made, each having six rabbits. FFSe-4 formulation was orally administered to Group I while Ricer ${ }^{\circledR}$ to Group II $\left(10 \mathrm{mg} \cdot \mathrm{kg}^{-1}\right)$. At various time intervals (0 to $24 \mathrm{hrs}$ ), blood samples $(0.5 \mathrm{ml})$ were collected and kept in tubes (heparinized). Plasma was separated by centrifugation and stored at $-20^{\circ} \mathrm{C}$ till further analysis.

2.6.2. Quantification of Plasma Concentration. Prepared plasma samples were analyzed for drug quantification by HPLC technique. Acetonitrile: Methanol: $(0.016 \mathrm{~mol} / \mathrm{l})$ Phosphoric Acid (10:10:80) were used as mobile phase (retention time: $3 \mathrm{~min}$, flow rate: $1 \mathrm{ml} / \mathrm{min}$ ). Reversed phase column (Supelco $\mathrm{C}_{18}, 25 \mathrm{~cm}$ in length, $4.6 \mathrm{~mm}$ width, and $5 \mu \mathrm{m}$ particle size), generally used for hydrophobic drugs, and precolumn (Supelco $\mathrm{C}_{18}$ ) were used at $37^{\circ} \mathrm{C}$. Prior to HPLC analysis, plasma samples were mixed with acetonitrile and then placed at $-20^{\circ} \mathrm{C}$ for 10 minutes followed by centrifugation to precipitate proteins. The supernatant $(20 \mu \mathrm{l})$ was then injected for the determination of FTD concentration using UV detector at $\lambda_{\max } 254 \mathrm{~nm}$ [33]. Famotidine concentration was determined from the area of chromatographic peak using the calibration curve.

2.6.3. Data Analysis. Different pharmacokinetic parameters were determined for non-compartmental model. Area under curve $\left(\mathrm{AUC}_{0 \rightarrow t}\right)$ was calculated from concentration-time curve by trapezoidal rule. From the individual plasma concentration-time curve, peak plasma concentration $\left(C_{\max }\right)$ and peak plasma concentration time $\left(T_{\max }\right)$ were calculated. Total area under the curve $\left(\mathrm{AUC}_{0 \rightarrow 24}\right)$ was determined by

$$
\mathrm{AUC}_{0 \rightarrow 24}=\mathrm{AUC}_{0 \rightarrow 24}+\frac{C_{t}}{K_{e}} .
$$

$C_{t}$ is FTD concentration at 24 th hour and $K_{e}$ is apparent elimination rate constant.

Relative bioavailability $\left(F_{r}\right)$ after 24 hours for equal dose was determined by

$$
F_{r}=\frac{\text { AUC-FFSe- } 4_{0 \rightarrow 24}}{\text { AUC-Marketed product } \text { pu }_{0 \rightarrow 24}} .
$$

One-way analysis of variance and $t$-test $(p<0.05)$ were used for statistical analysis of data.

\section{Results}

3.1. Dynamic Light Scattering. Unloaded SLNs were fabricated on the basis of three variable factors, that is, surfactant concentration, cosurfactant concentration, and magnetic stirring time. Significant changes were observed by changing these three variables (Figure 3). Best unloaded formulation was UFSe-11, having $z$-average particle size $127.8 \pm 2.3 \mathrm{~nm}$ and PDI $0.485 \pm 0.001$. Best drug loaded formulation was FFSe-4, having $z$-average particle size $111.9 \pm 1.3 \mathrm{~nm}, \mathrm{PDI} 0.464 \pm 0.03$, and zeta potential $-33.46 \pm 2 \mathrm{mV}$ (Figures 4 and 5).

3.2. Entrapment Efficiency and Drug Loading Capacity. Entrapment efficiency and drug loading capacity observed for FFSe- 1 formulation were $96 \pm 2.9 \%$ and $1.263 \pm 0.13 \%$ while for FFSe-5 formulation they were $59 \pm 3.17 \%$ and $3.375 \pm$ $0.18 \%$, respectively. The selected best formulation (FFSe-4) gave entrapment efficiency and drug loading capacity $84 \pm$ $2.7 \%$ and $2.709 \pm 0.13 \%$, respectively (Figure 6).

3.3. Drug-Excipients Interaction. Fourier transform infrared analysis is used specifically for assessing drug-excipients interaction in different formulations [35]. The major peaks of $\mathrm{C}=\mathrm{C}$ stretch at $1639 \mathrm{~cm}^{-1}, \mathrm{SO}_{2}$ stretch peak at $1147 \mathrm{~cm}^{-1}$, $\mathrm{C}-\mathrm{H}$ bend at $1284 \mathrm{~cm}^{-1}, \mathrm{C}=\mathrm{S}$ stretch at 1146 , and $\mathrm{N}-\mathrm{H}$ bend at $984 \mathrm{~cm}^{-1}$ were present in both unprocessed FTD and 


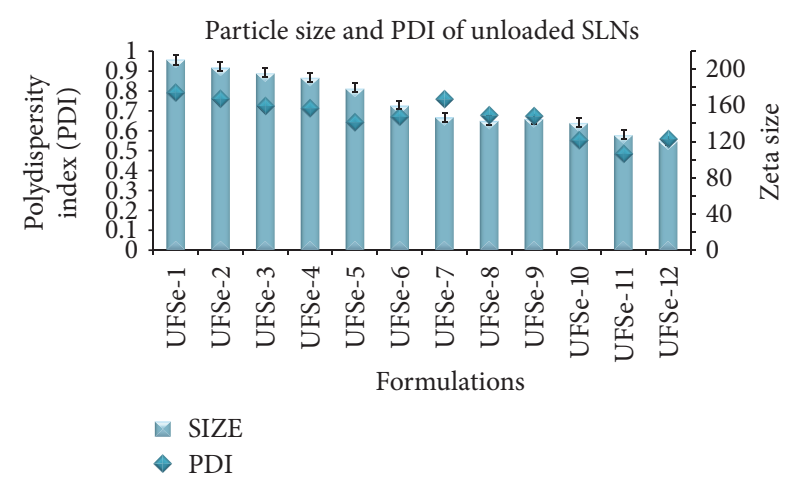

Figure 3: Particle size and PDI of unloaded SLNs formulations.

Number distribution data (\%)

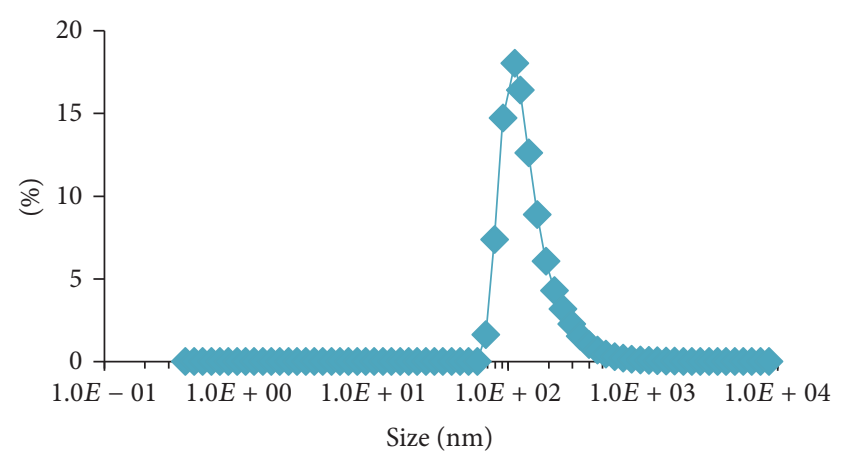

FIgURE 4: Particle size of FFSe-4.

processed FTD (FFSe-4). This clearly indicates no interaction between FTD and other excipients. The obtained spectra are shown (Figure 7).

3.4. Scanning Electron Microscopy (SEM). Shape and surface morphology of FFSe- 4 formulation was studied by SEM. SEM analysis showed solid and fairly spherical shaped particles with well-defined periphery. The particles size was also in nanometric range (Figure 8).

3.5. Powered X-Ray Diffraction (P-XRD). Unprocessed famotidine (FTD) showed a series of sharp peaks indicating its crystalline nature. In processed FTD (FFSe-4), most of these peaks were suppressed but few disappeared, indicating conversion to amorphous form (Figure 9).

3.6. Thermal Analysis. DSC thermograms of FTD (unprocessed), stearic acid (SA), physical mixture, and processed FTD (FFSe-4) were recorded separately. Sharp endothermic peak was observed for unprocessed FTD at $166.9^{\circ} \mathrm{C}$, SA at $69^{\circ} \mathrm{C}$, and physical mixture of FTD and SA at $166.5^{\circ} \mathrm{C}$ and $68.6^{\circ} \mathrm{C}$, respectively. Processed FTD (FFSe-4) showed endothermic peak at $160^{\circ} \mathrm{C}$ (Figure 10).

3.7. Stability Study. Processed FTD (FFSe-4) sample showed no significant change in particle size and PDI stored at refrigerated temperature $\left(5 \pm 2^{\circ} \mathrm{C}\right)$. Increase in particle size

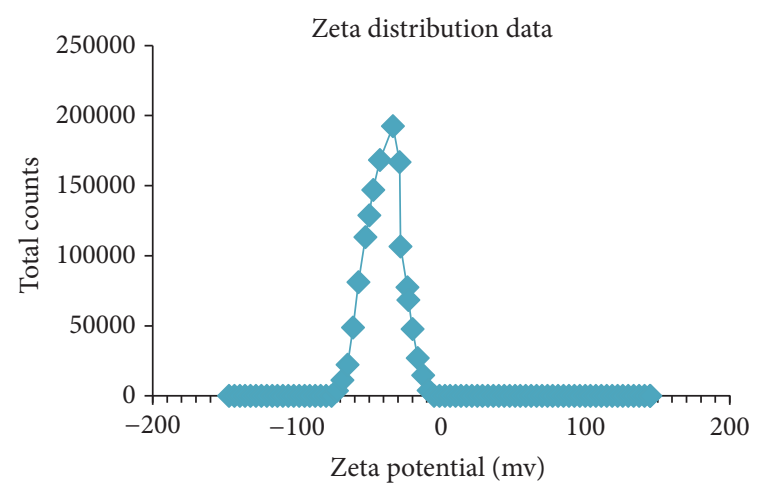

Figure 5: Zeta Potential of FFSe-4.

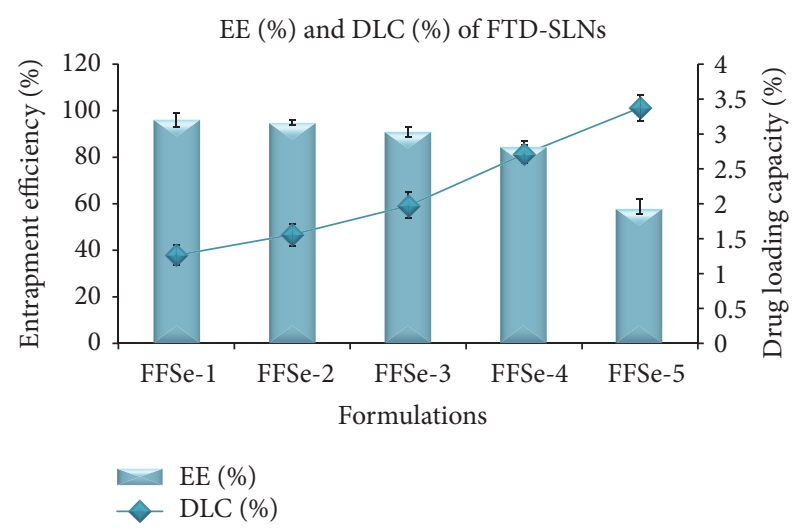

FIGURE 6: EE (\%) and DLC (\%) of different FTD-SLNs formulations.

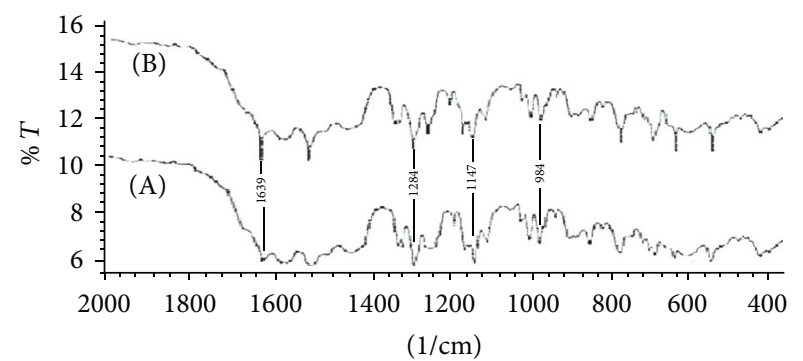

FIGURE 7: FT-IR spectra of unprocessed FTD (A) and processed FTD (FFSe-4) (B).

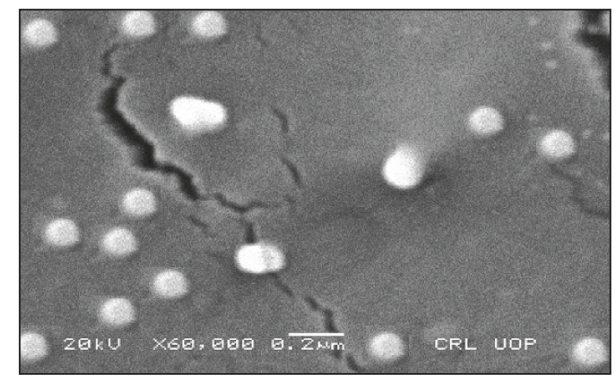

FIGURE 8: SEM micrograph of FFSe-4 formulation. 


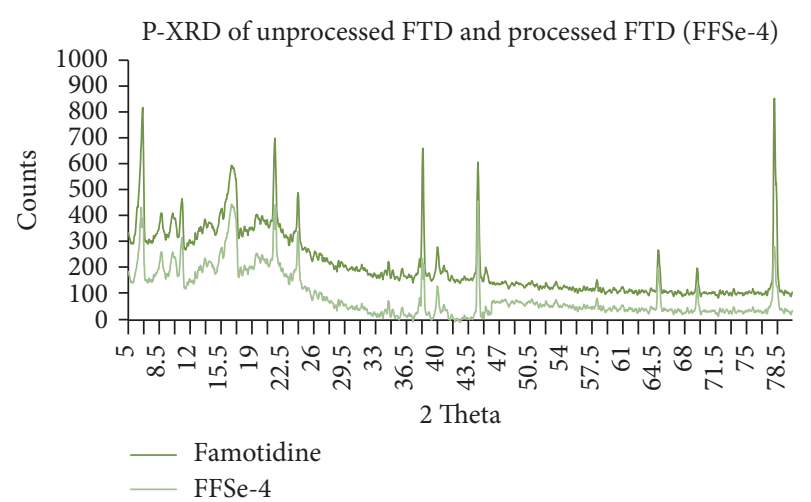

FIGURE 9: P-XRD of unprocessed FTD and processed FTD (FFSe-4).

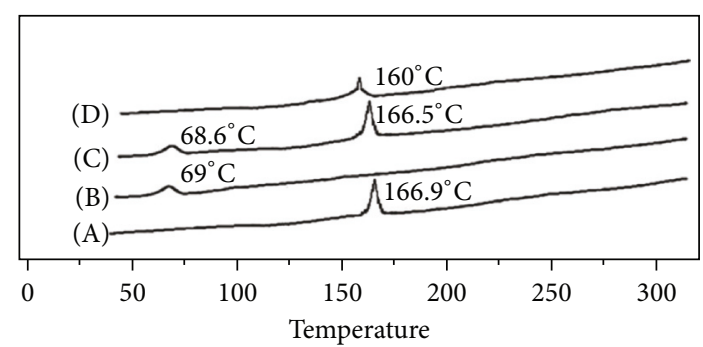

FIgURE 10: DSC of unprocessed FTD (A), stearic acid (B), physical mixture (C), and processed FTD (FFSe-4) (D).

TABLE 3: Cumulative percent release of FTD.

\begin{tabular}{lcccccc}
\hline S. No & $\begin{array}{c}\text { Time } \\
\text { (hrs) }\end{array}$ & \multicolumn{5}{c}{ Cumulative drug released (\%) } \\
\hline 1 & 0 & 0 & 0 & 0 & 0 & 0 \\
2 & 1 & 11.98 & 10.23 & 9.54 & 9.12 & 9.02 \\
3 & 2 & 24.63 & 20.57 & 20.21 & 16.86 & 15.23 \\
4 & 3 & 37.42 & 30.55 & 29.65 & 24.23 & 22.54 \\
5 & 4 & 47.87 & 41.34 & 38.25 & 32.12 & 28.61 \\
6 & 5 & 58.83 & 52.12 & 47.15 & 39.19 & 34.54 \\
7 & 6 & 67.82 & 62.11 & 54.58 & 45.98 & 40.84 \\
8 & 7 & 76.53 & 68.98 & 61.89 & 52.85 & 46.97 \\
9 & 8 & 83.12 & 76.34 & 70.12 & 59.12 & 52.87 \\
10 & 9 & 88.97 & 81.89 & 76.26 & 65.29 & 57.98 \\
11 & 10 & 93.24 & 88.12 & 82.32 & 70.84 & 63.74 \\
12 & 12 & 99.21 & 94.12 & 88.31 & 78.87 & 71.94 \\
\hline
\end{tabular}

at refrigerated temperature was less than $5 \%$ but at room temperature it was almost $15.91 \%$. At both temperatures, the particles growth was in acceptable range but PDI at room temperature exceeded the acceptable range (Figures 11 and 12). Statistically analyzed data from two tailed $t$-test showed $p$ value for particle size was 0.044 and PDI was 0.046 .

3.8. In Vitro Release of FTD from SLNS. During $12 \mathrm{hr}$ in vitro drug release study, cumulative percent drug release from FFSe- 1 to FFSe-5 formulations was $99.21 \%, 94.12 \%, 88.31 \%$, $78.87 \%$, and $71.94 \%$, respectively (Table 3 and Figure 13). FTD

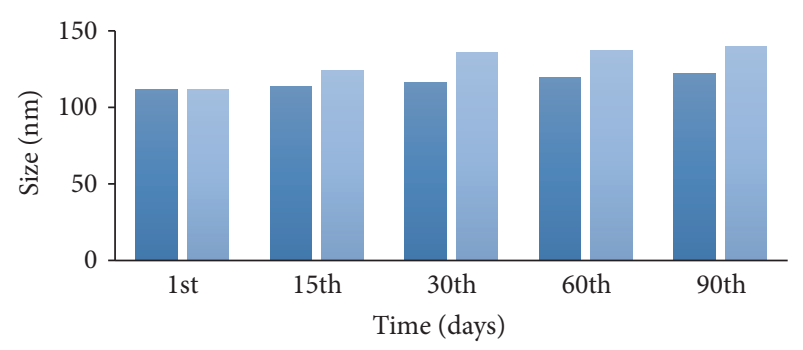

- Size (nm) at Refrigerator temperature

- Size $(\mathrm{nm})$ at room temperature

FIGURE 11: Particle size during stability study.

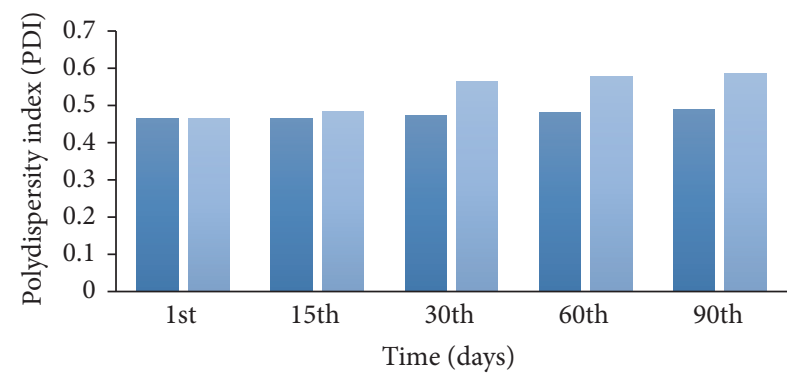

- PDI at refrigerator temperature

- PDI at room temperature

FIGURE 12: PDI during stability study.

release time from SLNs was directly proportional to drug pay load [23]. Further evaluation by putting the drug release data into different kinetic models showed that FTD loaded SLNs formulations followed zero order release kinetics with $R^{2}$ values in the range of $0.958-0.993$ [36]. However in Korsmeyer-Peppas model, release exponent was greater than $0.5(n>0.5)$ confirming non-Fickian diffusion kinetics for all formulations (Table 4) [37, 38].

3.9. In Vivo Pharmacokinetic Study. The plasma concentration-time curve of FFSe- 4 formulation and marketed product is shown (Figure 14) and pharmacokinetic parameters are also listed (Table 5). FTD plasma concentrations were significantly higher in rabbits treated with FFSe-4 than for those treated with marketed product.

Peak plasma concentration $\left(C_{\max }\right)$ for marketed product and FFSe-4 formulation was $0.498 \pm 0.14 \mu \mathrm{g} \cdot \mathrm{ml}^{-1}$ and $1.03 \pm 0.204 \mu \mathrm{g} \cdot \mathrm{ml}^{-1}$, respectively. $\mathrm{AUC}_{0 \rightarrow 24}$ for marketed product was $43.96 \mu \mathrm{g} \cdot \mathrm{hr} \cdot \mathrm{ml}^{-1}$ whereas for FFSe-4 was $231.22 \mu \mathrm{g} \cdot \mathrm{hr} \cdot \mathrm{ml}^{-1}$. FFSe-4 formulation showed 2.06fold increase in $C_{\max }$ and 5.25-fold increase in $\mathrm{AUC}_{0 \rightarrow 24}$ compared to marketed product. These results showed that FTD absorption was improved significantly in SLNs formulation compared with conventional dosage form (marketed product).

3.10. Discussion. Solvent emulsification evaporation (SEE) method has been used to fabricate FTD loaded SLNs. Optimized conditions for unloaded SLNs were stearic acid $(1.0 \mathrm{~g})$, 
TABLE 4: $R^{2}$ value of different kinetic models for FTD-SLNs formulation.

\begin{tabular}{lcccc}
\hline Formulations & Zero order $\left(R^{2}\right)$ & First order $\left(R^{2}\right)$ & Higuchi model $\left(R^{2}\right)$ & $\begin{array}{c}\text { Korsmeyer-Peppas model } \\
\text { Release exponent }(n)\end{array}$ \\
\hline FFSe-1 & 0.958 & 0.866 & 0.966 & 0.82067309 \\
FFSe-2 & 0.973 & 0.960 & 0.955 & 0.89488353 \\
FFSe-3 & 0.981 & 0.976 & 0.956 & 0.89479258 \\
FFSe-4 & 0.991 & 0.985 & 0.950 & 0.965 \\
FFSe-5 & 0.993 & 0.989 & 0.949 & 0.962 \\
\hline
\end{tabular}

TABLE 5: Pharmacokinetic parameters of FFSe-4 formulation and marketed product.

\begin{tabular}{|c|c|c|}
\hline Parameters & FFSe- 4 formulation & Marketed product \\
\hline$C_{\max }\left(\mu \mathrm{g} \mathrm{ml}^{-1}\right)$ & $1.03 \pm 0.204$ & $0.498 \pm 0.14$ \\
\hline$T_{\max }(\mathrm{h})$ & $12 \pm 0.2$ & $2 \pm 0.3$ \\
\hline $\operatorname{AUC}\left(\mu \mathrm{g} \cdot \mathrm{hr} \cdot \mathrm{ml}^{-1}\right)$ & $231.22 \pm 0.003$ & $43.96 \pm 0.021$ \\
\hline $\mathrm{Fr}$ & \multicolumn{2}{|c|}{5.25} \\
\hline
\end{tabular}

$(n=6, \bar{x} \pm \mathrm{SD})$.

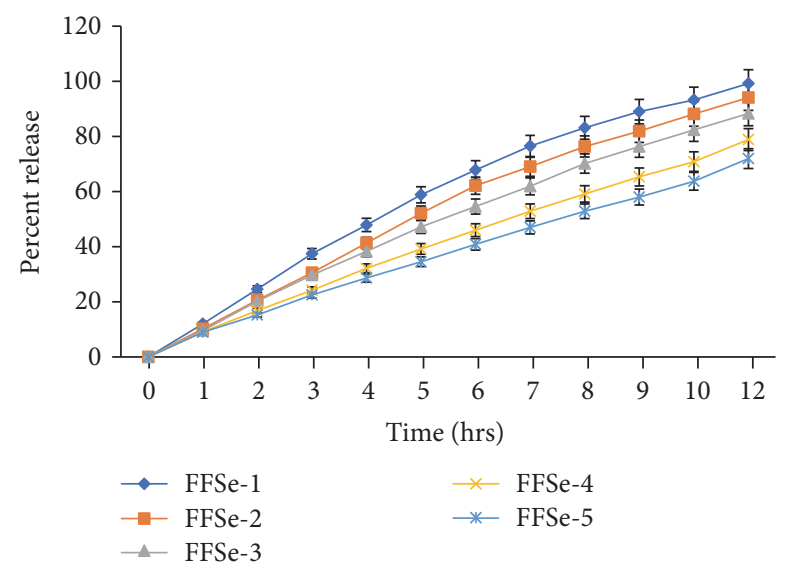

FIGURE 13: Drug release from different FTD-SLNs formulations.

Tween-80 (1.6 ml), PVP $(0.4 \mathrm{~g})$, and magnetic stirring time (15 minutes) (Table 1). $Z$-average particle size was reduced by increasing surfactant concentration (Tween-80); its higher concentration also gave better stability to small lipid droplets which prevent them from coalescence [39]. Addition of cosurfactant (PVP) further reduced $z$-average particle size as SLNs fabricated with surfactant/cosurfactant mixture have lower $z$-average particle size and better stability. PDI has been controlled and reduced by increasing magnetic stirring time as it has almost no effect on particle size reduction but only on PDI [40]. The optimized unloaded SLNs formulation (UFSe11) showed particle size $127.8 \pm 2.3 \mathrm{~nm}$. After drug (FTD) loading the particle size was reduced to $111.9 \pm 1.3 \mathrm{~nm}$ (FFSe4) having PDI $0.464 \pm 0.03$. After drug pay load, particle size reduced due to decreased free lipid content [41]. Zeta potential of FFSe- 4 formulation was $-33.46 \pm 2 \mathrm{mV}$, sufficient for electrostatic stability [42].

The PDI $<0.5$ and zeta potential \pm 30 revealed that the fabricated nanodispersion would be stable in nature [43].

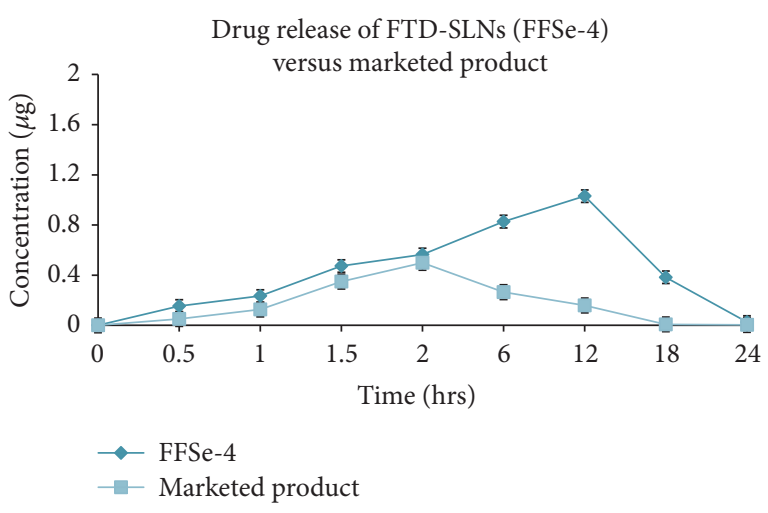

FIGURE 14: In vivo drug release from FFSe-4 formulation versus marketed product.

Both of these values for FTD-SLNs were within the range, exhibiting electrostatic stabilization having no aggregation which led to preventing Ostwald ripening and particle growth [42].

The formulation (FFSe-4) gave entrapment efficiency and drug loading capacity $84 \pm 2.7 \%$ and $2.709 \pm 0.13 \%$, respectively, with maximum encapsulation and higher drug loading efficiency. It has been reported that in polymer and lipid based nanoparticulate drug delivery systems, the binding energy of the drugs with the polymers and lipids plays a key role in successful encapsulation of drugs [44]. In this case, it might be attributed to the high binding energy of the FTD with stearic acid, tween-80, and PVP which results in maximum entrapment efficacy and drug loading capacity.

However, EE\% decreased from $96 \%$ to $59 \%$ as FTD pay load increased from $40 \mathrm{mg}$ (FFSe-1) to $200 \mathrm{mg}$ (FFSe-5). This sudden fall in EE\% might be due to loading of FTD beyond saturation level of lipid [23]. Lipophilic drugs can gain super-saturation in melted lipids; on cooling, saturation level reduces and excessive quantity of drug tends to partition in outer shell or external solvent [5].

FT-IR spectra of unprocessed FTD and processed FTD (FFSe-4 formulation) confirm the compatibility of FTD with the formulation components (Figure 7). Scanning electron microscopy further confirmed nanometric size particles of SLNs loaded with FTD. Micrograph of SEM (Figure 8) shows solid, identical, and fairly spherical shaped particles with a well-defined periphery. Most of the SLNs are present in dispersed form with homogeneous distribution which exhibit amorphous nature of the produced nanoparticles. P$\mathrm{XRD}$ studies also confirmed the amorphous nature of the 
FTD loaded SLNs, as the disappearance and reduction in intensities of the peaks are indicative for amorphous nature of the particles (Figure 9) $[43,45]$. DSC studies confirmed the amorphous nature of the FTD loaded SLNs, because for unprocessed FTD, sharp melting point peak appeared on $166.6^{\circ} \mathrm{C}$ while for FTD loaded SLNs formulation it was $160^{\circ} \mathrm{C}$ (Figure 10 ). This small diffused peak indicated reduced particle size of FTD, enlarged surface area, and closed contact between solid lipid (stearic acid) and drug (FTD) which could be considered for the change of FTD from crystalline to amorphous state $[46,47]$.

In comparison with room temperature, refrigerated temperature was best for the stability of FFSe- 4 formulation. Three-month study showed no significant change in size and PDI of the sample when stored at refrigerated temperature (Figure 11). However, at room temperature some growth was observed for the initial 30 days which is because of the amorphous nature of the FTD-SLNs followed by stabilization for rest of the period. Additionally, at room temperature, amorphous solids have increased free energy which results in decreased stability $[48,49]$.

In vitro study showed that increased payload of FTD resulted in prolonged drug release time (Figure 13) [23]. Release of FTD from SLNs followed zero order kinetics. However Korsmeyer-Peppas model showed that the release exponent $(n)$ was greater than 0.5 which confirmed nonFickian diffusion kinetics for all SLNs formulations [37, 38].

The interesting results obtained from statistically analyzed data of in vivo pharmacokinetics confirmed boosted oral bioavailability with sustained release profile of FTDSLNs (FFSe-4) compared to marketed product (Table 5). SLNs as drug delivery system open angles to formulate already available drugs (BCS-II and BCS-IV) in the market to boost their oral bioavailability and attain sustained release behavior. SLNs are not only responsible for improvement of oral absorption but can correspondingly be formulated for parenteral administration, which need additional studies [50].

\section{Conclusion}

This research work concluded that various processing parameters are the characteristic key factors to prepare appropriate lipid carriers for efficient loading of the selected drug. SLNs have been surfaced as novel drug carriers for famotidine with boosted oral bioavailability and strong sustained drug release performance. We have exposed that famotidine in form of SLNs is an encouraging nanomedicine with valueadded physical stability and prolonged release profile. Also, there was good affinity found between famotidine and stearic acid. In vitro and in vivo release study confirmed that SLNs system is very suitable to improve oral delivery of poor water soluble drug like famotidine with increased solubility and permeability which in turn enhanced bioavailability. In future perspectives, the produced FTD loaded SLNs could potentially be transformed into solid dosage form followed by in vitro and in vivo assessments.
Thus, it is concluded that sustained release FTD-SLNs were successfully fabricated by simple and reproducible technique (solvent emulsification-evaporation method) which has potential to be scaled up for commercial production and no sophisticated instrument is required during fabrication.

\section{Conflicts of Interest}

The authors report no conflicts of interest in this research.

\section{Acknowledgments}

The authors would like to acknowledge Polyfine Chempharma (Pvt) Ltd (Peshawar-Pakistan) for providing generous gift of famotidine and Ferozsons Laboratories limited, Nowshera, Pakistan, for providing FT-IR facilities.

\section{References}

[1] Y. Kawabata, K. Wada, M. Nakatani, S. Yamada, and S. Onoue, "Formulation design for poorly water-soluble drugs based on biopharmaceutics classification system: basic approaches and practical applications," International Journal of Pharmaceutics, vol. 420, no. 1, pp. 1-10, 2011.

[2] S. Das, W. K. Ng, P. Kanaujia, S. Kim, and R. B. H. Tan, "Formulation design, preparation and physicochemical characterizations of solid lipid nanoparticles containing a hydrophobic drug: Effects of process variables," Colloids and Surfaces B: Biointerfaces, vol. 88, no. 1, pp. 483-489, 2011.

[3] H. Harde, M. Das, and S. Jain, "Solid lipid nanoparticles: An oral bioavailability enhancer vehicle," Expert Opinion on Drug Delivery, vol. 8, no. 11, pp. 1407-1424, 2011.

[4] B. Sarmento, S. Martins, D. Ferreira, and E. B. Souto, "Oral insulin delivery by means of solid lipid nanoparticles," International Journal of Nanomedicine, vol. 2, no. 4, pp. 743-749, 2007.

[5] R. H. Müller, K. Mäder, and S. Gohla, "Solid lipid nanoparticles (SLN) for controlled drug delivery-a review of the state of the art," European Journal of Pharmaceutics and Biopharmaceutics, vol. 50, no. 1, pp. 161-177, 2000.

[6] W. Mehnert and K. Mäder, "Solid lipid nanoparticles: production, characterization and applications," Advanced Drug Delivery Reviews, vol. 47, no. 2-3, pp. 165-196, 2001.

[7] R. Shah, D. Eldridge, E. Palombo, and I. Harding, "Optimisation and stability assessment of solid lipid nanoparticles using particle size and zeta potential," Journal of Physical Science, vol. 25, no. 1, pp. 59-75, 2014.

[8] H. A. Ebrahimi, Y. Javadzadeh, M. Hamidi, and M. B. Jalali, "Repaglinide-loaded solid lipid nanoparticles: effect of using different surfactants/stabilizers on physicochemical properties of nanoparticles," DARU Journal of Pharmaceutical Sciences, vol. 23, no. 1, article 46, 2015.

[9] C. Vitorino, F. A. Carvalho, A. J. Almeida, J. J. Sousa, and A. A. C. C. Pais, "The size of solid lipid nanoparticles: An interpretation from experimental design," Colloids and Surfaces B: Biointerfaces, vol. 84, no. 1, pp. 117-130, 2011.

[10] Y. Kinoshita, T. Hashimoto, A. Kawamura et al., "Effects of famotidine, mosapride and tandospirone for treatment of functional dyspepsia," Alimentary Pharmacology and Therapeutics, Supplement, vol. 21, no. s2, pp. 37-41, 2005.

[11] D. P. Patel, R. R. Shah, A. P. Patel, and P. K. Tank, "Development and validation of first order derivative uv-spectroscopic 
method for estimation of ibuprofen and famotidine in synthetic mixture," Pharma science Monitor, vol. 3, no. 4, 2012.

[12] R. M. O. Aman, M. M. O. Meshali, and G. M. A. Abdelghani, "Ion-exchange complex of famotidine: sustained release and taste masking approach of stable liquid dosage form," Drug Discoveries \& Therapeutics, vol. 8, no. 6, pp. 268-275, 2014.

[13] F. M. Mady, A. E. Abou-Taleb, K. A. Khaled et al., "Evaluation of carboxymethyl- $\beta$-cyclodextrin with acid function: Improvement of chemical stability, oral bioavailability and bitter taste of famotidine," International Journal of Pharmaceutics, vol. 397, no. 1-2, pp. 1-8, 2010.

[14] F. M. Mady, A. E. Abou-Taleb, K. A. Khaled et al., "Enhancement of the aqueous solubility and masking the bitter taste of famotidine using drug/SBE- $\beta$-CyD/Povidone K30 complexation approach," Journal of Pharmaceutical Sciences, vol. 99, no. 10, pp. 4285-4294, 2010.

[15] D. J. Patel and J. K. Patel, "Design and evaluation of famotidine mucoadhesive nanoparticles for aspirin induced ulcer treatment," Brazilian Archives of Biology and Technology, vol. 56, no. 2, pp. 223-236, 2013

[16] A. Avdeef, C. M. Berger, and C. Brownell, "pH-metric solubility. 2: correlation between the acid-base titration and the saturation shake-flask solubility-pH methods," Pharmaceutical Research, vol. 17, no. 1, pp. 85-89, 2000.

[17] K. C. Yeh, A. N. Chremos, J. H. Lin et al., "Single-dose pharmacokinetics and bioavailability of famotidine in man. Results of multicenter collaborative studies," Biopharmaceutics \& Drug Disposition, vol. 8, no. 6, pp. 549-560, 1987.

[18] T. Takabatake, H. Ohta, M. Maekawa et al., "Pharmacokinetics of famotidine, a new H2-receptor antagonist, in relation to renal function," European Journal of Clinical Pharmacology, vol. 28, no. 3, pp. 327-331, 1985.

[19] S. A. Wissing, O. Kayser, and R. H. Müller, "Solid lipid nanoparticles for parenteral drug delivery," Advanced Drug Delivery Reviews, vol. 56, no. 9, pp. 1257-1272, 2004.

[20] M. Abbaspour, B. S. Makhmalzadeh, Z. Arastoo, A. Jahangiri, and R. Shiralipour, "Effect of anionic polymers on drug loading and release from clindamycin phosphate solid lipid nanoparticles," Tropical Journal of Pharmaceutical Research, vol. 12, no. 4, pp. 477-482, 2013.

[21] K.-H. Song, S.-J. Chung, and C.-K. Shim, "Enhanced intestinal absorption of salmon calcitonin ( $\mathrm{sCT}$ ) from proliposomes containing bile salts," Journal of Controlled Release, vol. 106, no. 3, pp. 298-308, 2005.

[22] N. Venkatesan, K. Uchino, K. Amagase, Y. Ito, N. Shibata, and K. Takada, "Gastro-intestinal patch system for the delivery of erythropoietin," Journal of Controlled Release, vol. 111, no. 1-2, pp. 19-26, 2006.

[23] M. Rehman, A. Madni, A. Ihsan et al., "Solid and liquid lipid-based binary solid lipid nanoparticles of diacerein: In vitro evaluation of sustained release, simultaneous loading of gold nanoparticles, and potential thermoresponsive behavior," International Journal of Nanomedicine, vol. 10, pp. 2805-2814, 2015.

[24] B. Ozturk, S. Argin, M. Ozilgen, and D. J. McClements, "Formation and stabilization of nanoemulsion-based vitamin e delivery systems using natural surfactants: Quillaja saponin and lecithin," Journal of Food Engineering, vol. 142, pp. 57-63, 2014.

[25] W. Abdelwahed, G. Degobert, S. Stainmesse, and H. Fessi, "Freeze-drying of nanoparticles: formulation, process and storage considerations," Advanced Drug Delivery Reviews, vol. 58, no. 15, pp. 1688-1713, 2006.
[26] B. Tiţa, A. Fuliaş, G. Bandur, E. Marian, and D. Tiţa, "Compatibility study between ketoprofen and pharmaceutical excipients used in solid dosage forms," Journal of Pharmaceutical and Biomedical Analysis, vol. 56, no. 2, pp. 221-227, 2011.

[27] S. Uprit, R. K. Sahu, A. Roy, and A. Pare, "Preparation and characterization of minoxidil loaded nanostructured lipid carrier gel for effective treatment of alopecia," Saudi Pharmaceutical Journal, vol. 21, pp. 379-385, 2013.

[28] A. Dubes, H. Parrot-Lopez, W. Abdelwahed et al., "Scanning electron microscopy and atomic force microscopy imaging of solid lipid nanoparticles derived from amphiphilic cyclodextrins," European Journal of Pharmaceutics and Biopharmaceutics, vol. 55, no. 3, pp. 279-282, 2003.

[29] C. Racault, F. Langlais, and R. Naslain, "Solid-state synthesis and characterization of the ternary phase Ti3SiC2," Journal of Materials Science, vol. 29, no. 13, pp. 3384-3392, 1994.

[30] D. Hou, C. Xie, K. Huang, and C. Zhu, "The production and characteristics of solid lipid nanoparticles (SLNs)," Biomaterials, vol. 24, no. 10, pp. 1781-1785, 2003.

[31] A. del Pozo-Rodríguez, M. A. Solinís, A. R. Gascón, and J. L. Pedraz, "Short- and long-term stability study of lyophilized solid lipid nanoparticles for gene therapy," European Journal of Pharmaceutics and Biopharmaceutics, vol. 71, no. 2, pp. 181-189, 2009.

[32] U. Bhardwaj and D. J. Burgess, "A novel USP apparatus 4 based release testing method for dispersed systems," International Journal of Pharmaceutics, vol. 388, no. 1-2, pp. 287-294, 2010.

[33] A. C. Moffat, M. D. Osselton, B. Widdop, and L. Y. Galichet, "Clarke's analysis of drugs and poisons," 2004.

[34] Roohullah, Z. Iqbal, F. Nasir et al., "Sustained release carbamezapine matrix tablets prepared by solvent-evaporation technique using different polymers," Middle East Journal of Scientific Research, vol. 15, no. 10, pp. 1368-1374, 2013.

[35] F. Barboza, D. D. Vecchia, M. P. Tagliari, M. A. S. Silva, and H. K. Stulzer, "Differential scanning calorimetry as a screening technique in compatibility studies of acyclovir extended release formulations," Pharmaceutical Chemistry Journal, vol. 43, no. 6, pp. 363-368, 2009.

[36] P. Costa and J. M. Sousa Lobo, "Modeling and comparison of dissolution profiles," European Journal of Pharmaceutical Sciences, vol. 13, no. 2, pp. 123-133, 2001.

[37] A. A. Sadiq and A. Abdul Rassol, "Formulation and evaluation of silibinin loaded solid lipid nanoparticles for peroral use targeting lower part of gastrointestinal tract," Int J Pharm PharmSci, vol. 6, no. 1, pp. 55-67, 2014.

[38] M. Barzegar-Jalali, "Kinetic analysis of drug release from nanoparticles," Journal of Pharmacy Pharmaceutical Sciences, vol. 11, no. 1, pp. 167-177, 2008.

[39] A. Kovacevic, S. Savic, G. Vuleta, R. H. Müller, and C. M. Keck, "Polyhydroxy surfactants for the formulation of lipid nanoparticles (SLN and NLC): effects on size, physical stability and particle matrix structure," International Journal of Pharmaceutics, vol. 406, no. 1-2, pp. 163-172, 2011.

[40] H. Baharifar, G. Tavoosidana, R. Karimi et al., "Optimization of self-assembled chitosan/streptokinase nanoparticles and evaluation of their cytotoxicity and thrombolytic activity," Journal of Nanoscience and Nanotechnology, vol. 15, no. 12, pp. 10127-10133, 2015.

[41] P. P. Kumar, P. Gayatri, R. Sunil, S. Jagamohan, and Y. M. Rao, "Atorvastatin loaded solidlipid nanoparticles: formulation, optimization, and in vitro characterization," IOSR Journal of Pharmacy, vol. 2, no. 5, pp. 23-32, 2012. 
[42] J. Liu, W. Hu, H. Chen, Q. Ni, H. Xu, and X. Yang, "Isotretinoinloaded solid lipid nanoparticles with skin targeting for topical delivery," International Journal of Pharmaceutics, vol. 328, no. 2, pp. 191-195, 2007.

[43] H. S. M. Ali, P. York, A. M. A. Ali, and N. Blagden, "Hydrocortisone nanosuspensions for ophthalmic delivery: A comparative study between microfluidic nanoprecipitation and wet milling," Journal of Controlled Release, vol. 149, no. 2, pp. 175-181, 2011.

[44] Y. Liu, J. Pan, and S.-S. Feng, "Nanoparticles of lipid monolayer shell and biodegradable polymer core for controlled release of paclitaxel: Effects of surfactants on particles size, characteristics and in vitro performance," International Journal of Pharmaceutics, vol. 395, no. 1-2, pp. 243-250, 2010.

[45] S. Khan, M. D. Matas, J. Zhang, and J. Anwar, "Nanocrystal preparation: low-energy precipitation method revisited," Crystal Growth and Design, vol. 13, no. 7, pp. 2766-2777, 2013.

[46] E. S. Farboud, S. A. Nasrollahi, and Z. Tabbakhi, "Novel formulation and evaluation of a Q10-loaded solid lipid nanoparticle cream: in vitro and in vivo studies," International Journal of Nanomedicine, vol. 6, pp. 611-617, 2011.

[47] J. Y. Fang, C. L. Fang, C. H. Liu, and Y. H. Su, "Lipid nanoparticles as vehicles for topical psoralen delivery: solid lipid nanoparticles (SLN) versus nanostructured lipid carriers (NLC)," European Journal of Pharmaceutics and Biopharmaceutics, vol. 70, no. 2, pp. 633-640, 2008.

[48] A. Khawam and D. R. Flanagan, "Basics and applications of solid-state kinetics: a pharmaceutical perspective," Journal of Pharmaceutical Sciences, vol. 95, no. 3, pp. 472-498, 2006.

[49] B. C. Hancock and G. Zografi, "Characteristics and significance of the amorphous state in pharmaceutical systems," Journal of Pharmaceutical Sciences, vol. 86, no. 1, pp. 1-12, 1997.

[50] S. C. Yang, L. F. Lu, Y. Cai, J. B. Zhu, B. W. Liang, and C. Z. Yang, "Body distribution in mice of intravenously injected camptothecin solid lipid nanoparticles and targeting effect on brain," Journal of Controlled Release, vol. 59, no. 3, pp. 299-307, 1999. 

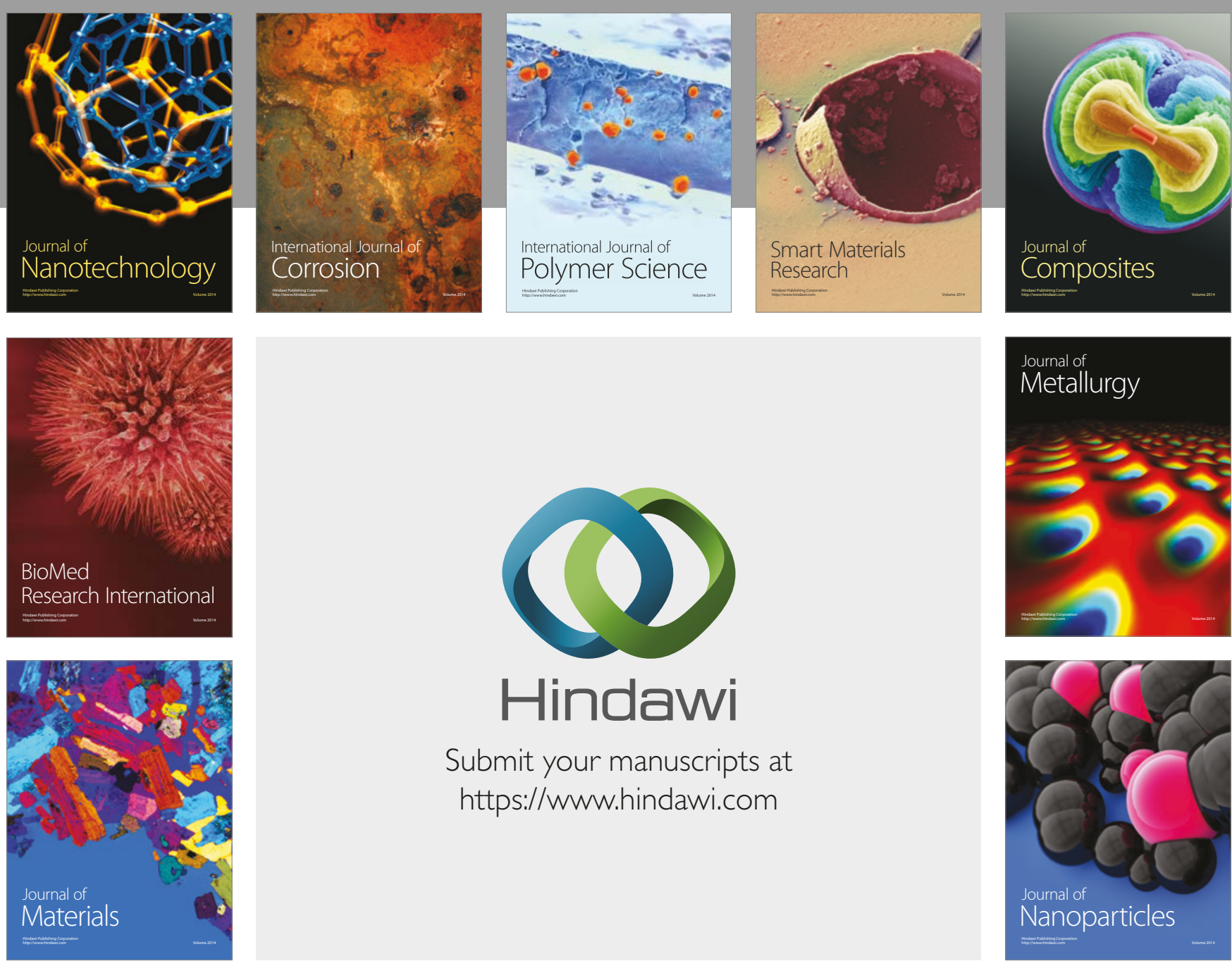

\section{Hindawi}

Submit your manuscripts at

https://www.hindawi.com
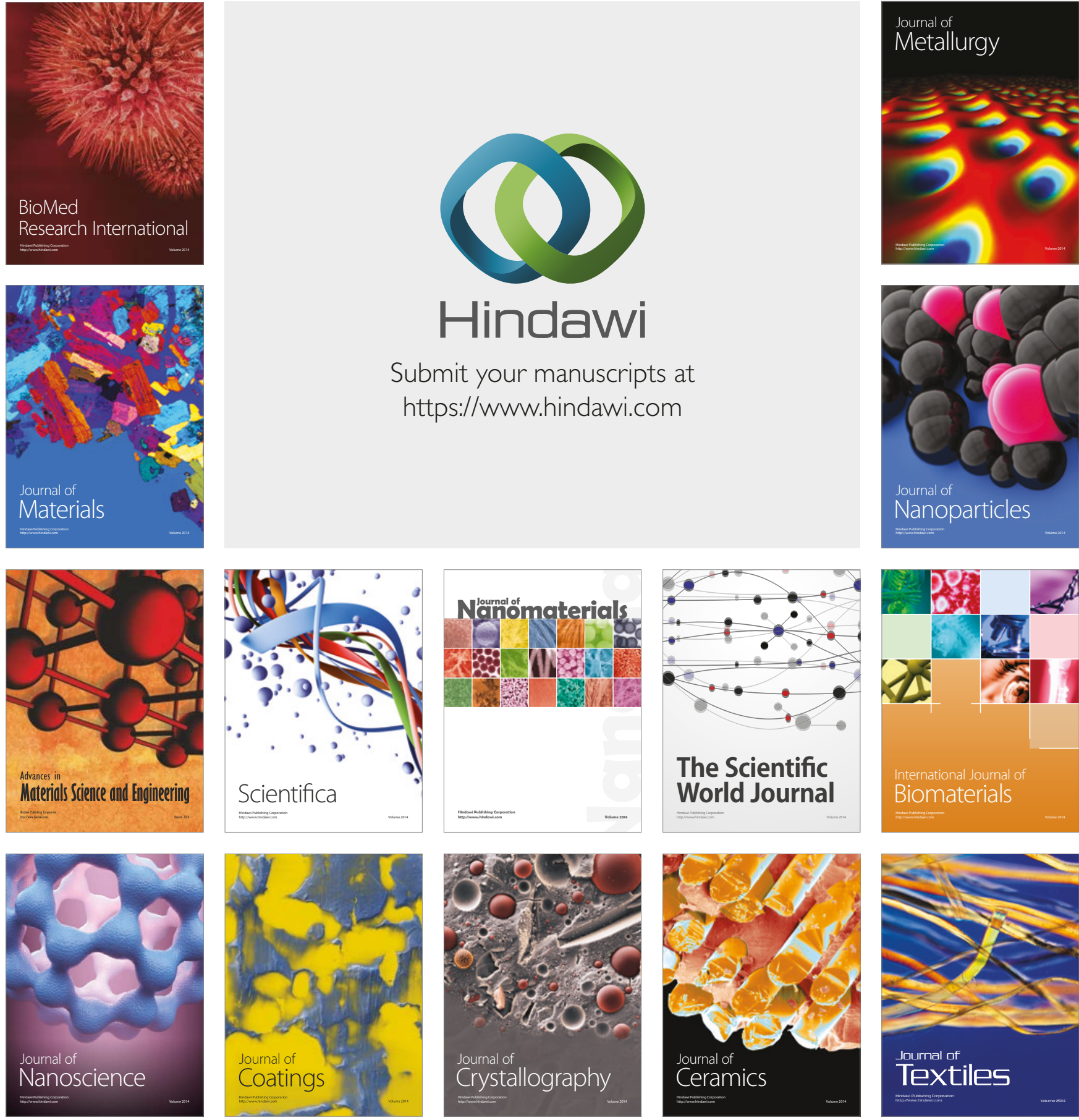

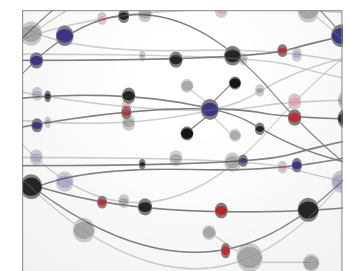

The Scientific World Journal
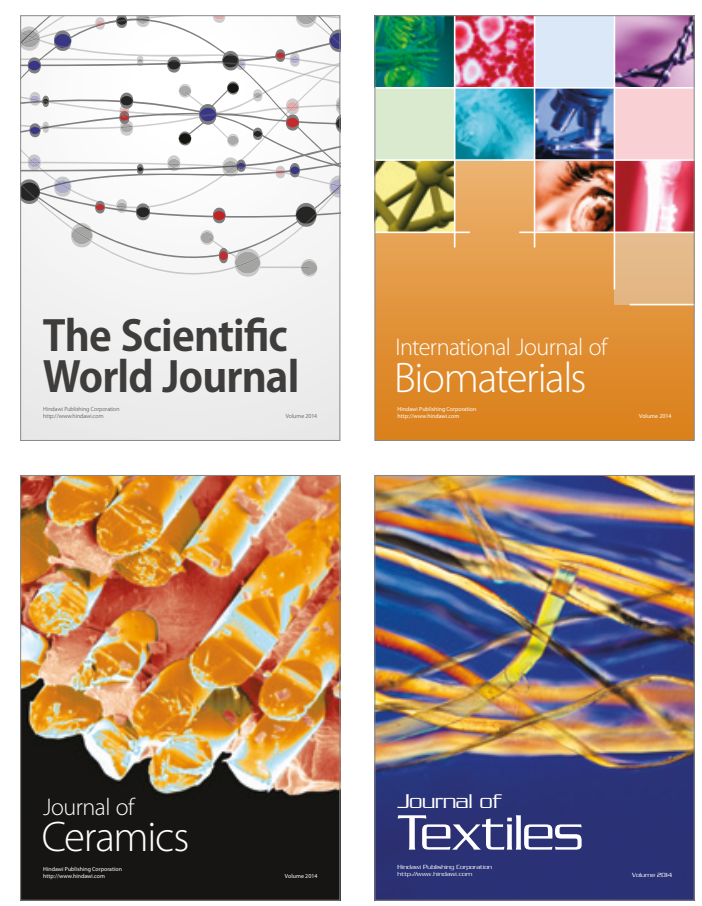